\title{
Allogeneic hematopoietic stem cell transplantation from an alternative stem cell source in Fanconi anemia patients: analysis of 47 patients from a single institution
}

\section{C.R. de Medeiros ${ }^{1}$, M.A. Bitencourt ${ }^{1}$, J. Zanis-Neto ${ }^{1}$, E.C.P. Maluf, D.S. Carvalho ${ }^{3}$, C.S. Bonfim ${ }^{1}$, V.M. Funke ${ }^{1}$, D.C. Setubal ${ }^{1}$, N. Farah ${ }^{4}$ and R. Pasquini ${ }^{1}$}

\author{
${ }^{1}$ Serviço de Transplante de Medula Óssea, \\ 2Departamento de Clínica Médica, \\ ${ }^{3}$ Departamento de Saúde Comunitária, \\ ${ }^{4}$ Laboratório de Imunogenética e Biologia Molecular, \\ Hospital de Clínicas, Universidade Federal do Paraná, \\ Curitiba, PR, Brasil
}

Correspondence

C.R. de Medeiros

Serviço de Transplante de

Medula Óssea

Hospital de Clínicas, UFPR

Rua General Carneiro, 181, 15o andar 80060-900 Curitiba, PR

Brasil

Fax: +55-41-336-1110

E-mail: crdemedeiros@terra.com.br

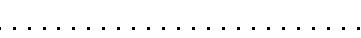

Received October 27, 2005

Accepted July 7, 2006

\begin{abstract}
We transplanted 47 patients with Fanconi anemia using an alternative source of hematopoietic cells. The patients were assigned to the following groups: group 1, unrelated bone marrow $(\mathrm{N}=15)$; group 2, unrelated cord blood $(\mathrm{N}=17)$, and group 3 , related non-sibling bone marrow $(\mathrm{N}=15)$. Twenty-four patients $(51 \%)$ had complete engraftment, which was not influenced by gender $(P=0.87)$, age $(P=0.45)$, dose of cyclophosphamide ( $\mathrm{P}=0.80)$, nucleated cell dose infused ( $\mathrm{P}$ $=0.60)$, or use of anti-T serotherapy $(P=0.20)$. Favorable factors for superior engraftment were full HLA compatibility (independent of the source of cells; $\mathrm{P}=0.007)$ and use of a fludarabine-based conditioning regimen $(P=0.046)$. Unfavorable factors were $\geq 25$ transfusions pretransplant $(\mathrm{P}=0.011)$ and degree of HLA disparity $(\mathrm{P}=0.007)$. Intensity of mucositis $(\mathrm{P}=0.50)$ and use of androgen prior to transplant had no influence on survival $(\mathrm{P}=0.80)$. Acute graft-versus-host disease (GVHD) grade II-IV and chronic GVHD were diagnosed in 47 and $23 \%$ of available patients, respectively, and infections prevailed as the main cause of death, associated or not with GVHD. Eighteen patients are alive, the Kaplan-Meyer overall survival is $38 \%$ at $\sim 8$ years, and the best results were obtained with related non-sibling bone marrow patients. Three recommendations emerged from the present study: fludarabine as part of conditioning, transplant in patients with $<25$ transfusions and avoidance of HLA disparity. In addition, an extended family search (even when consanguinity is not present) seeking for a related non-sibling donor is highly recommended.
\end{abstract}

Key words

- Fanconi anemia

- Transplant in Fanconi

anemia

- Alternative donors

- Allogeneic hematopoietic stem cell transplantation in

Fanconi anemia

- Alternative stem cell source 


\section{Introduction}

In addition to having chromosomal instability and malformations, Fanconi anemia (FA) patients also have a tendency to marrow failure and the risk of developing acute myeloid leukemia, myelodysplastic syndromes and epithelial malignancies later in life (1). Allogeneic hematopoietic stem cell transplantation (HSCT) is currently the only way to restore normal hematopoiesis, improving the survival of these patients (2). Unfortunately only $\sim 25 \%$ have an available compatible sibling donor, a fact that has led to a search for alternative hematopoietic stem cell (HSC) sources. These sources have included matched or partially matched relatives, unrelated volunteer donor bone marrow, and related or unrelated cord blood.

We have previously reported the encouraging results of HSCT in FA patients from a compatible matched sibling donor, using progressively lower doses of cyclophosphamide during the conditioning regimen (3-5). Other single institution and multicenter studies using cyclophosphamide alone or associated with radiotherapy have also reported similar good results, with $>60 \%$ probability of long-term survival $(2,6,7)$. However, when alternative donors are used, higher rates of graft failure, transplant-related toxicity and graft-versus-host disease (GVHD) are observed and long-term survival is $\sim 30 \%$ (7).

We report here a retrospective analysis of our experience with 47 FA patients transplanted with HSC from an alternative donor, with emphasis on factors predicting the outcome.

\section{Patients and Methods}

\section{Patients}

We transplanted 134 patients with FA from March 1983 to August 2004. Fortyseven of these 134 FA patients received HSC from an alternative donor between
August 1992 and August 2004. All patients were in the severe or moderately aplastic phase of the illness, with no clinical or hematologic evidence of leukemia or myelodysplasia. Diagnosis was based on clinical features of FA and diepoxybutane-induced chromosomal breakage. The complementation groups were analyzed in 36 patients, but had been defined in only 20 by August 2004, with 13 belonging to FANC A, 4 to FANC $\mathrm{G}$, and 3 to FANC C. All related donors were tested before transplant and none showed evidence of FA.

The protocol was approved by the Ethics Committee of the Hospital de Clínicas and all patients or parents gave written informed consent. Typing of both donor and recipient pairs involved low resolution for HLA-A and HLA-B by reverse sequence specific oligonucleotides using Luminex technology (One Lambda Cat. RSSO1A/RSSO1B, Canoga Park, CA, USA), and high resolution for DRB1 using sequence specific primers (One Lambda Cat. SSP2H). Unrelated bone marrow and cord blood were identified and obtained through the National Marrow Donor Program, Caitlyn Raymond International Registry, New York Blood Center, and Brazilian Marrow Donor Registry. The patients were divided into three groups according to the source of HSC: group 1, unrelated bone marrow (UBM, $\mathrm{N}=15$ ); group 2, unrelated cord blood (UCB, $\mathrm{N}=17$ ), and group 3 , related non-sibling bone marrow (RnsBM, $\mathrm{N}=15$ ). In the RnsBM group, consanguinity was observed in the families of 5 patients, with great-grand parents being cousins in 1 , grandparents in 2, and parents in 2 families. No consanguinity was present in 7 patient families. The presence or absence of consanguinity could not be determined in 3 patient families. Information about patients is presented in Table 1.

\section{Methods}

Conditioning regimens are listed in Table 
2. Patients were isolated in single or double rooms with high efficiency particulate air filters. In case of ABO incompatibility, red blood cells or plasma were removed by starch sedimentation. Patients seronegative for $\mathrm{cy}$ tomegalovirus (CMV) were transfused with CMV-negative blood or leukodepleted blood. Acyclovir prophylaxis for herpes virus and trimethopim-sulfamethoxazole for Pneumocystis carinii were used in all patients along with fluconazole for fungus prevention. Weekly antigenemia for monitoring CMV viremia and preemptive treatment with ganciclovir were the routine. Details about GVHD prevention are given in Table 2 . Acute GVHD (aGVHD) grade II to IV was defined using published criteria (12) and patients surviving $>21$ days post-transplant with engraftment were considered to be at risk. Patients engrafted and surviving $>70$ days were at risk for chronic GVHD (cGVHD) (13). Neutrophil engraftment is defined as an absolute neutrophil count $>0.5$ x $10^{9} / \mathrm{L}$ for at least 2 consecutive days without treatment with a growth factor. Time to platelet engraftment is defined as the first of three consecutive days on which the platelet count was higher than $50 \times 10^{9} / \mathrm{L}$ without transfusion for 7 days. Documented marrow donor engraftment was confirmed by $\mathrm{ABO}$ typing in patients with major red blood cell group differences, by chromosomal analysis for sex-mismatched patient/donor pairs and by PCR targeting variable number of tandem repeat loci.

\section{Statistical analysis}

Odds-ratios and $95 \%$ confidence interval were calculated to estimate the association of engraftment with the studied factors. The evaluation included univariate analysis and unconditional logistic regression considering the following variables: age at the time of bone marrow transplantation $(<10$ years and $\geq 10$ years), HLA compatibility (none, one or two mismatches), number of transfu- sions prior to transplant $(<25$ transfusions and $\geq 25$ transfusions), number of nucleated cells infused (median as cutoff), gender, and use of fludarabine, anti-serotherapy and other conditioning regimens. The variables in-

Table 1. Pre-transplant features of 47 patients.

\begin{tabular}{lccc}
\hline & Group 1 & Group 2 & Group 3 \\
\hline No. of patients & 15 & 17 & 15 \\
Gender (male:female) & $9: 6$ & $5: 12$ & $8: 7$ \\
Age, years (median) & $7-19(11)$ & $5-14(8)$ & $3-31(8)$ \\
No. of transfusion (median) & $0-400(41)$ & $1-101(29)$ & $2-61(24)$ \\
No. of cells (median) & $2.3-19.3 \times 10^{8} / \mathrm{kg}$ & $2.7-19.2 \times 10^{7} / \mathrm{kg}$ & $1.9-8.8 \times 10^{8} / \mathrm{kg}$ \\
& $\left(4.8 \times 10^{8} / \mathrm{kg}\right)$ & $\left(5.2 \times 10^{7} / \mathrm{kg}\right)$ & $\left(4.6 \times 10^{8} / \mathrm{kg}\right)$ \\
HLA match & & & 8 \\
6/6 & 12 & 2 & 3 \\
5/6 & 3 & & 3 \\
$\quad$ B & & 3 & 1 \\
$\quad$ A & & & \\
$\quad$ DRB1 & & 7 & 5 \\
4/6 & & 1 & \\
$\quad$ A,B & & 7 & \\
$\quad$ B,DRB1 & 2 & & \\
Previous TX & & & \\
\hline
\end{tabular}

No. of transfusions = number of packed red cells and platelets given before hematopoietic stem cell transplantation (HSCT); No. of cells = nucleated cell dose infused; 6 / 6: full match; 5/6: 1 mismatch (in B, A or DRB1); 4/6: 2 mismatches (in A and B or B and DRB1); previous TX = use of an androgen prior to HSCT.

Table 2. Characteristics of the transplant procedures.

\begin{tabular}{|c|c|c|}
\hline & No. of patients & References \\
\hline \multicolumn{3}{|l|}{ Conditioning regimens } \\
\hline CFA $^{140}$ & 6 & 3 \\
\hline $\mathrm{CFA}^{60}$ & 12 & 5 \\
\hline$C F A^{60}+F L U+A T S$ & 11 & 8 \\
\hline $\mathrm{CFA}{ }^{40}+\mathrm{ATS}+\mathrm{TBI}$ & 2 & 9 \\
\hline FLU + TBI & 4 & 10 \\
\hline $\mathrm{CFA}^{40}+\mathrm{TBI}$ & 2 & 9 \\
\hline $\mathrm{CFA}^{60}+\mathrm{FLU}$ & 7 & 11 \\
\hline $\mathrm{CFA}^{100}+$ ATS & 2 & 9 \\
\hline$C F A^{60}+B u+A T S$ & 1 & 9 \\
\hline \multicolumn{3}{|c|}{ Graft-versus-host disease prophylaxis } \\
\hline CSA + MTX & 33 & 12 \\
\hline CSA alone & 8 & 9 \\
\hline $\mathrm{CSA}+\mathrm{MMf}$ & 4 & 10 \\
\hline$C S A+T C D$ & 2 & 9 \\
\hline
\end{tabular}

Superscript numbers indicate the total dose of cyclophosphamide in $\mathrm{mg} / \mathrm{kg}$ of patient weight; CFA = cyclophosphamide; FLU = fludarabine; $\mathrm{ATS}$ = anti-T serotherapy; TBI = total body irradiation; $\mathrm{Bu}=$ busulfan; $\mathrm{CSA}=$ cyclosporine; $\mathrm{MTX}=$ methotrexate; $\mathrm{MMf}=$ mycophenolate mofetil; TCD $=$ T-cell depletion. 
cluded in the model were chosen if the $\mathrm{P}$ value was less than 0.20 , or when the variable was considered to be important for the model (14). We used the Stata 8 software to perform forward multivariate analysis. Estimates of survival were calculated by the Kaplan-Meier product limit method (15) using the Graph Pad Prism software.

\section{Results}

\section{Engraftment}

The median time to granulocytic engraftment was 18 days (range: 10 to 58 days), and the median time to platelet recovery was 23 days (range: 15 to 64 days). Five patients died before time to engraftment could be determined ( 2 in the UCB group and 3 in the RnsBM group), and 6 engrafted partially (only neutrophil engraftment, 2 in the UBM group, 3 in the UCB group and 1 in the RnsBM group). Twenty-four patients (51\%) had complete hematopoietic recovery ( 7 in the UBM group, 7 in the UCB group and 10 in the RnsBM group). Factors that might influence engraftment were analyzed. These included age, gender, nucleated cell dose infused, HLA compatibility, transfusion prior to transplant, and conditioning regimen used (specifically

\begin{tabular}{|c|c|c|c|c|c|c|}
\hline \multirow[t]{2}{*}{ Studied factors } & \multicolumn{3}{|c|}{ Univariate } & \multicolumn{3}{|c|}{ Multivariate } \\
\hline & OR & $95 \% \mathrm{Cl}$ & $P$ & OR & $95 \% \mathrm{Cl}$ & $P$ \\
\hline HLA compatibility & 2.43 & $1.13-5.21$ & 0.02 & 4.90 & $1.56-15.45$ & 0.007 \\
\hline Prior transfusion $(\geq 25)$ & 4.55 & $1.20-17.27$ & 0.01 & 7.21 & $1.59-32.78$ & 0.011 \\
\hline Fludarabine (yes) & 0.77 & $0.24-2.46$ & 0.66 & 0.16 & $0.03-0.96$ & 0.046 \\
\hline Other conditioning regimen & 1.47 & $0.29-7.61$ & 0.64 & 4.90 & $0.64-37.58$ & 0.13 \\
\hline Nucleated cells infused (>median) & 0.32 & $0.09-1.13$ & 0.06 & 0.38 & $0.10-1.46$ & 0.16 \\
\hline Other immunosuppression & 2.36 & $0.37-14.60$ & 0.36 & 4.20 & $0.51-34.83$ & 0.18 \\
\hline Anti-serotherapy & 0.86 & $0.23-3.13$ & 0.82 & 0.43 & $0.08-2.35$ & 0.33 \\
\hline Age at bone marrow transplantation & 1.29 & $0.40-4.12$ & 0.67 & 1.96 & $0.34-11.41$ & 0.45 \\
\hline Methotrexate & 0.54 & $0.16-1.86$ & 0.31 & 0.70 & $0.16-3.09$ & 0.65 \\
\hline Gender & 0.76 & $0.24-2.46$ & 0.66 & 0.89 & $0.22-3.64$ & 0.87 \\
\hline
\end{tabular}

$\mathrm{OR}=$ odds ratio; $95 \% \mathrm{Cl}=95 \%$ confidence interval. dose of cyclophosphamide and use of anti-T serotherapy and/or fludarabine).

Statistical analysis (Table 3) demonstrated that gender $(\mathrm{P}=0.87)$, age $(\mathrm{P}=0.45)$, dose of cyclophosphamide $(\mathrm{P}=0.80)$, nucleated cell dose infused $(\mathrm{P}=0.16)$, and use of anti-T serotherapy had no influence on engraftment $(\mathrm{P}=0.20)$. The same statistical analysis showed that favorable factors for superior engraftment were HLA full compatibility independent of the source of cells $(P=0.007)$, as well as fludarabine as part of the conditioning regimen $(\mathrm{P}=0.046)$. Unfavorable factors were $\geq 25$ transfusions prior to transplant $(\mathrm{P}=0.01)$ and degree of HLA disparity $(\mathrm{P}=0.007)$.

\section{Transplant-related toxicity}

Five patients were considered to be infected before HSCT and 2 survived. Intensity of mucositis had no influence on survival, since 23 patients had grade 0 to II and 24 patients grade III to IV, and 12 and 17 died, respectively $(\mathrm{P}=0.50)$. The use of an androgen prior to transplantation also had no influence on survival $(\mathrm{P}=0.80)$.

aGVHD grade II-IV was diagnosed in 14 of 30 patients (47\%) and cGVHD in 5 of 22 available patients (23\%). The source of HSC had no influence on the development of $\operatorname{aGVHD}(\mathrm{P}=0.80)$ and cGVHD $(\mathrm{P}=0.90)$.

Infections were commonly encountered among these patients, prevailing as the main cause of death, associated or not with GVHD. We also observed the incidence of concomitant infections. Among the UBM, UCB and RnsBM groups, death occurred between days 31 and 217 (median: 57 days), days 7 and 256 (median: 58 days) and days 8 and 98 (median: 45 days), respectively.

\section{Survival}

Overall, 18 of the 47 patients described here are alive with a median post-transplant follow-up of 18 months (range: 6-97 months). 
The Kaplan-Meier estimate of survival was $38 \%$ at $\sim 8$ years (Figure 1). Complete HLA compatibility influenced survival $(\mathrm{P}=0.04)$, as also did pre-transplant transfusion $(\mathrm{P}=$ 0.04) (Figures 2 and 3, respectively). The degree of HLA mismatch and respective survival for all patients are illustrated in Figure 4. There was a survival benefit when comparing the fully matched RnsBM group with the mismatched UCB group $(\mathrm{P}=0.04)$, the fully matched UBM group $(\mathrm{P}=0.04)$, the mismatched RnsBM group ( $\mathrm{P}=0.009)$, the mismatched UBM group $(\mathrm{P}=0.001)$, and the UCB group with two mismatches $(\mathrm{P}=$ $0.05)$.

\section{Discussion}

Since the HSCT is the only way to restore normal hematopoiesis and improve survival of FA patients with hematological manifestation of the disease, efforts have been made to search for a methodology capable of adequate engraftment associated with low toxicity. When a compatible matched sibling donor is available, survival has improved from a few survivors in the early 80's (16) to an almost 90\% survival rate in 2003 (17). Lower doses of alkylating agents associated or not with low doses of radiotherapy, use of fludarabine and anti-T serotherapy, along with new approaches to GVHD prevention and superior resources of support therapy, have significantly contributed to the improved survival of FA patients. Unfortunately, these benefits favor only $25 \%$ of FA patients, while the other $\sim 75 \%$ undergoing HSCT from an alternative donor still face a high incidence of graft rejection and transplant-related toxicity, which drastically affect their survival.

Graft failure (GF) was the main factor directly involved in the poor survival of our patients. Twenty-three patients (49\%) had only partial or no engraftment, dying shortly after the transplant. Of 24 patients with hematopoietic recovery, 18 remain alive. We retrospectively analyzed the risk factors involved in GF. HLA incompatibility and degree of disparity, $\geq 25$ transfusions prior to transplantation and absence of fludarabine in the conditioning regimen had an unfavorable influence on engraftment. In a retrospective multicenter study, Guardiola et al.

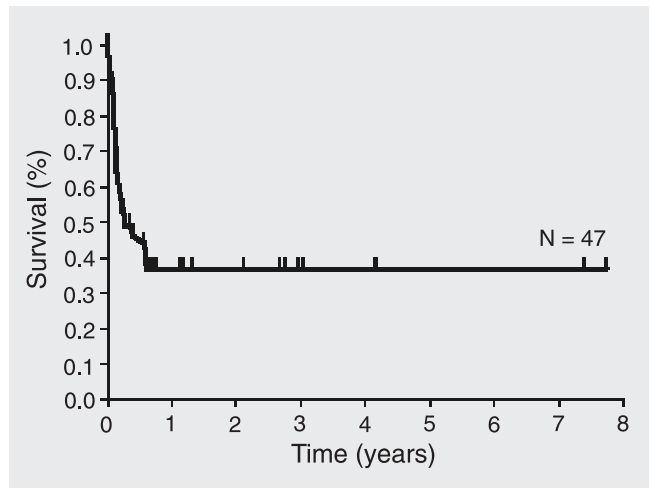

Figure 1. Probability of survival.

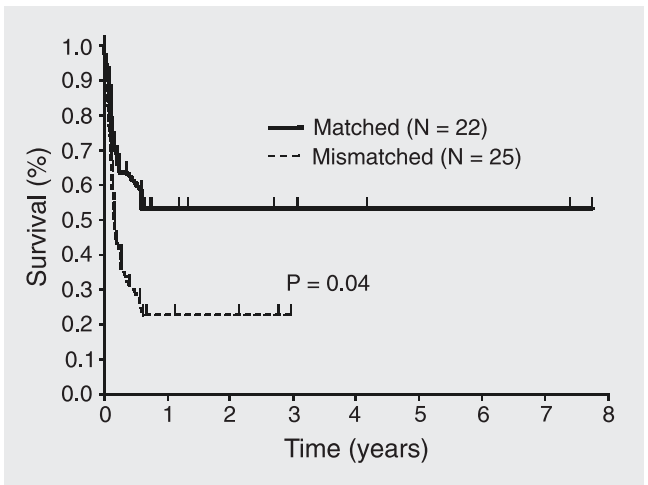

Figure 2. Probability of survival according to HLA-compatibility $(P=0.04$, log-rank test $)$.

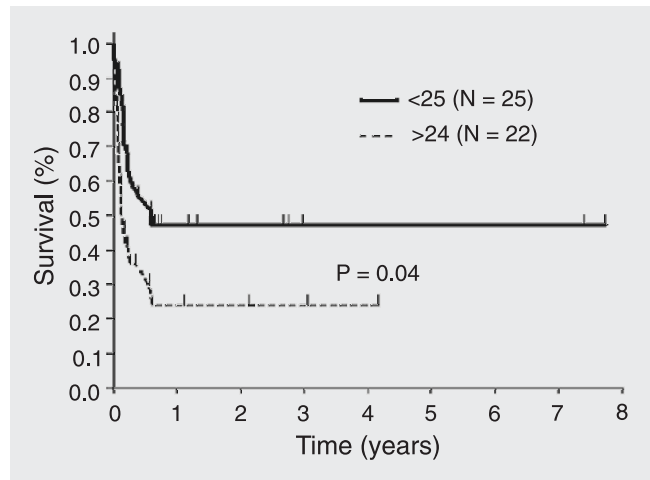

Figure 3. Probability of survival according to number of transfusions prior to transplant $(P=0.04$, log-rank test). 
(7) reported that 17 and $31 \%$ of patients had no granulocyte or platelet recovery, respectively, after marrow transplant from an unrelated donor, and the probability of secondary graft failure was $19 \%$ at 1 year. GF was noted more frequently when female donors were used, mainly because the nucleated cell dose infused was smaller when compared with grafts from male donors, a fact not observed in our analysis. In a retrospective multicenter study of 72 unrelated cord blood transplants in FA, Gluckman et al. (18) reported GF in $46 \%$ of patients. Unfavorable factors were number of HLA differences $>2$ antigens and nucleated cell dose $<4.4 \times 10^{7} / \mathrm{kg}$, while fludarabine had a positive impact on engraftment. In a study of 31 patients, MacMillan et al. (19) obtained 100\% primary neutrophil engraftment, but patients considered at high risk (age $>18$ years, HLA mismatched and a high degree of disparity, advanced phase of disease with myelodysplastic syndrome or acute myelogenous leukemia, and previous bacterial or fungal infections) had a $33 \%$ probability of survival at 2 years against $81 \%$ for standard risk

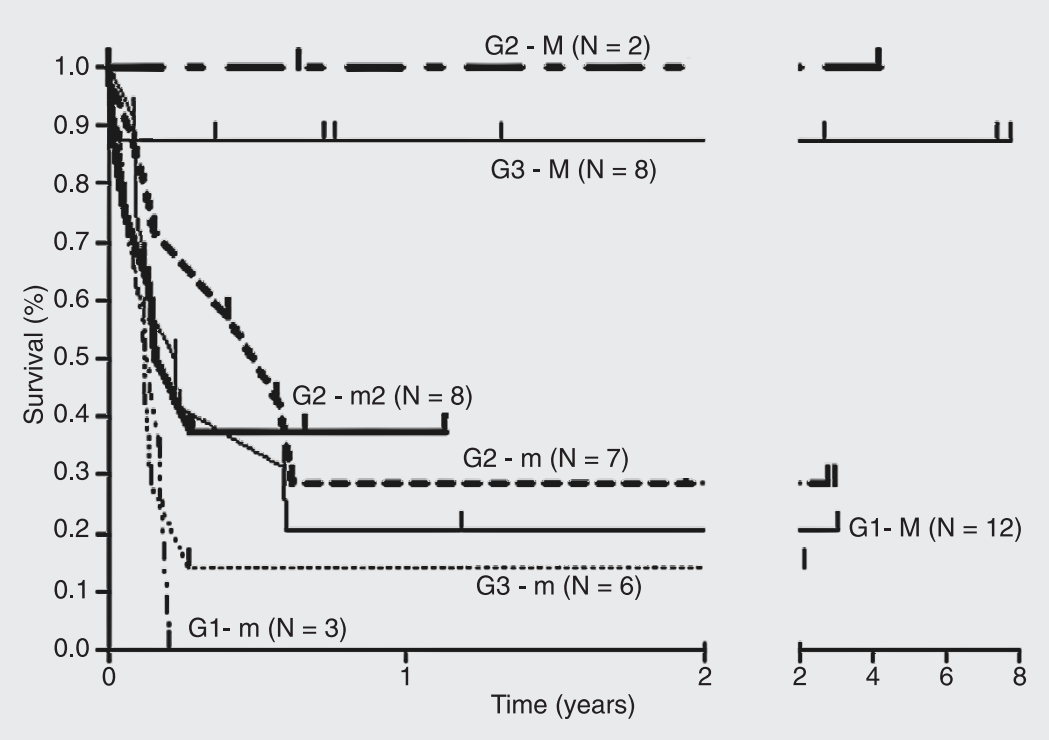

Figure 4. Probability of survival according to group and degree of compatibility. G1 = group 1, unrelated bone marrow patients; G2 = group 2, unrelated cord blood patients; $\mathrm{G} 3$ = group 3 , related non-sibling bone marrow patients; $M=$ matched; $m=$ mismatched; $m 2=2 / 6$ mismatches; $\mathrm{N}=$ number of patients. patients. Since all patients received fludarabine, it was not possible to analyze the influence of fludarabine on survival. In our analysis, age and previous infection had no influence on outcome. Recently, Wagner et al. (11) examined 98 patients receiving a transplant from an unrelated marrow donor, with $46 \%$ receiving non-fludarabine-based preparative regimens. Fludarabine positively influenced neutrophil and platelet recovery, with consequent lower mortality. The corresponding probability of overall survival was 57 vs $17 \%$ in the non-fludarabine group. These investigators also observed a significantly higher mortality in patients receiving $>20$ blood product transfusions prior to transplantation. Thus, we conclude from these studies that a small number of transfusions pre-transplant, lesser HLA differences and fludarabine-based conditioning regimen are important for the outcome of FA patients.

Transplant-related mortality was closely related to engraftment. Twenty-three (49\%) of 47 patients died between days +7 and +226 (median: 45 days). Five died very early, before engraftment, and only 4 died after day +100 . A second transplant was provided to 7 patients with primary GF, but engraftment failed again. All of these patients were severely immunosuppressed, a fact favoring opportunistic infections by concomitant agents. The finding of disseminated toxoplasmosis in four patients was clear evidence of their severe immunosuppression (20). In addition, in states of iron overload, free iron catalyzes the conversion of reactive oxygen species, such as superoxide anion and hydrogen peroxide, to highly toxic free radicals, potentially increasing the risk of bacterial, fungal and other opportunistic infections (21). We observed aGVHD grade $\geq$ II in 14 cases (47\%), and 12 had grade IIIIV. Literature data regarding unrelated donor transplantation in FA patients have shown an incidence of aGVHD $\geq$ II ranging from 17 to $55 \%(6,17,19,22)$ with recipients of $\mathrm{T}$ cell-depleted grafts having a lower incidence 
of aGVHD. Boyer et al. (23) using highly purified CD34+ peripheral blood stem cells for transplant with alternative donors in 8 FA patients, noticed only 2 cases of aGVHD of the skin, and no development of II-IV aGVHD or cGVHD. Nine of our patients died as a consequence of aGVHD. cGVHD was observed in $4(18 \%)$ of 22 available patients, and previous reports demonstrated a cGVHD incidence of 11 to $18 \%$, with Tcell-depleted and cord blood grafts being favorable factors $(7,11,18,22)$.

In an effort to overcome the barrier of rejection, we have tried to intensify the conditioning regimen, but, as observed by MacMillan et al. (24), more intensive conditioning and post-transplant immunosuppression have not improved engraftment and have probably increased GVHD due to the release of cytokines secondary to damage and activation of host tissues, including intestinal mucosa and liver.

Our best outcomes were obtained when the source of HSC was a related non-sibling donor. When comparing the three groups of patients, there was a survival advantage for the RnsBM group over the UCB group $(\mathrm{P}=$ $0.01)$ and UBM group $(P=0.05)$. Guardiola et al. (7) reported similar transplant results whether the graft was from an HLA-matched pheno-identical related donor or from an HLA geno-identical sibling donor. However, in the presence of HLA incompatibility, the 2 -year survival was dismal $(\sim 6 \%)$. In contrast, Harris (17) reported that, even with a fully matched phenotypically identical relative donor, the higher risk of graft rejection and GVHD recommends the use of a conditioning regimen suited for unrelated donors. When searching for an HSC donor, if a sibling donor is not available, our routine has been to look for a suitable donor among aunts, uncles, cousins, and grandparents. In our extended family search we studied 263 families and found a suitable donor for 45 patients (17\%). Twenty-four had a full-match compatible relative donor, 17 had a one mismatch donor in class I, and 4 patients had a one mismatch donor in class II. A German study (25) showed that only $4 \%$ of pediatric patients with hematological malignancies had an HLA-acceptable donor after an extended family search. Nevertheless, studies enrolling Arabic families with consanguinity demonstrated that an HLA-matched donor was found by an extended family search for $\sim 60 \%$ of the patients (26). Thus, an extended family search may be worthwhile, particularly in distinct ethnic populations. In general, the larger the size of the extended family, the more useful the search for identical donors will be (27). Besides, consanguinity is not a pre-requisite for donor procurement, as observed in the present study.

When transplanting FA patients with HSC from an alternative stem cell source, overcoming the barrier of rejection with less transplant toxicity is crucial. Efforts must be directed towards tailored regimens, and three recommendations emerge: fludarabine as part of the conditioning regimen, transplant for patients with $<25$ transfusions and use of the closest HLA matched selection. Also, in conformity with our results, an extended family search seeking a relative donor is highly recommended.

\section{Acknowledgments}

The authors are indebted to Miss Heliz R. Neves, our data manager, for her excellent contribution. 


\section{References}

1. Young NS, Alter BP. Clinical features of Fanconi's anemia. In: Young NS, Alter BP (Editors), Aplastic anemia acquired and inherited. Philadelphia: Saunders; 1994. p 275-309.

2. Gluckman E, Auerbach AD, Horowitz MM, Sobocinski KA, Ash RC, Bortin MM, et al. Bone marrow transplantation for Fanconi anemia. Blood 1995; 86: 2856-2862.

3. Zanis-Neto J, Ribeiro RC, Medeiros C, Andrade RJ, Ogasawara V, Hush $\mathrm{M}$, et al. Bone marrow transplantation for patients with Fanconi anemia: a study of 24 cases from a single institution. Bone Marrow Transplant 1995; 15: 293-298.

4. Medeiros CR, Zanis-Neto J, Pasquini R. Bone marrow transplantation for patients with Fanconi anemia: reduced doses of cyclophosphamide without irradiation as conditioning. Bone Marrow Transplant 1999; 24: 849-852.

5. Zanis-Neto J, Flowers ME, Medeiros CR, Bitencourt MA, Bonfim CM, Setubal DC, et al. Low-dose cyclophosphamide conditioning for haematopoietic cell transplantation from HLA-matched related donors in patients with Fanconi anaemia. Br J Haematol 2005; 130: 99106.

6. Socie G, Devergie A, Girinski T, Piel G, Ribaud P, Esperou H, et al. Transplantation for Fanconi's anaemia: long-term follow-up of fifty patients transplanted from a sibling donor after low-dose cyclophosphamide and thoraco-abdominal irradiation for conditioning. $\mathrm{Br} J$ Haematol 1998; 103: 249-255.

7. Guardiola P, Socie G, Pasquini R, Dokal I, Ortega JJ, van WeelSipman $M$, et al. Allogeneic stem cell transplantation for Fanconi anaemia. Severe Aplastic Anaemia Working Party of the EBMT and EUFAR. European Group for Blood and Marrow Transplantation. Bone Marrow Transplant 1998; 21 (Suppl 2): S24-S27.

8. Yabe H, Inoue H, Shimizu T, Yasuda Y, Matsumoto M, Hattori K, et al. Improved result of unrelated donor bone marrow transplantation for Fanconi anemia. Blood 2002; 100 (Suppl 2): 466b (Abstract 5442).

9. Zanis-Neto J. Transplante de medula óssea em anemia de Fanconi escalonando doses decrescentes de ciclofosfamida. [PhD's thesis]. Curitiba: Universidade Federal do Paraná; 1999.

10. de Medeiros CR, Silva LM, Pasquini R. Unrelated cord blood transplantation in a Fanconi anemia patient using fludarabine-based conditioning. Bone Marrow Transplant 2001; 28: 110-112.

11. Wagner JE, Eapen M, Harris RE, MacMillan ML, Auerbach AD. Unrelated donor transplantation for Fanconi anemia: analysis of prognostic factors impacting engraftment and survival. Blood 2004; 104 (Suppl 1): 235a (Abstract 824).

12. Glucksberg $H$, Storb $R$, Fefer A, Buckner CD, Neiman PE, Clift RA, et al. Clinical manifestations of graft-versus-host disease in human recipients of marrow from HL-A-matched sibling donors. Transplantation 1974; 18: 295-304.

13. Atkinson K, Horowitz MM, Gale RP, van Bekkum DW, Gluckman E, Good RA, et al. Risk factors for chronic graft-versus-host disease after HLA-identical sibling bone marrow transplantation. Blood 1990; 75: 2459-2464.

14. Hennekens $\mathrm{CH}$, Buring JB. Analysis of epidemiological studies: evaluating the role of confounding. In: Mayrent SL (Editor), Epidemiology in medicine. Boston, Toronto: Little, Brown and Company; 1987. p 287-323.

15. Kaplan EL, Meier P. Non parametric estimation from incomplete observations. J Am Stat Assoc 1958; 53: 457-481.

16. Berger R, Bernheim A, Gluckman E, Gisselbrecht C. In vitro effect of cyclophosphamide metabolites on chromosomes of Fanconi anaemia patients. Br J Haematol 1980; 45: 565-568.

17. Harris RE. Fanconi anemia: Matched sibling donor hematopoietic cell transplantation. In: Joyce Owen J, Frohnmayer L, Eiler ME (Editors), Fanconi anemia: standards for clinical care. Eugene: Fanconi Anemia Research Fund; 2003. p 95-106.

18. Gluckman E, Rocha V, Ionescu I, Pasquini R, Harris R, Wagner J, et al. Results of unrelated cord blood transplants in Fanconi anemia. Blood 2004; 104 (Suppl 1): 590a (Abstract 2145).

19. MacMillan ML, Weisdorf DJ, Slungaard A, Defor TE, Champagne $M A$, Auerbach $A D$, et al. High probability of survival in standard risk patients with Fanconi anemia after alternate donor hematopoietic cell transplantation. Blood 2002; 100 (Suppl 1): 857a (Abstract 3382).

20. de Medeiros BC, de Medeiros CR, Werner B, Loddo G, Pasquini R, Bleggi-Torres LF. Disseminated toxoplasmosis after bone marrow transplantation: report of 9 cases. Transpl Infect Dis 2001; 3: 24-28.

21. Evens AM, Mehta J, Gordon LI. Rust and corrosion in hematopoietic stem cell transplantation: the problem of iron and oxidative stress. Bone Marrow Transplant 2004; 34: 561-571.

22. Guardiola P, Pasquini R, Dokal I, Ortega JJ, van Weel-Sipman M, Marsh JC, et al. Outcome of 69 allogeneic stem cell transplantations for Fanconi anemia using HLA-matched unrelated donors: a study on behalf of the European Group for Blood and Marrow Transplantation. Blood 2000; 95: 422-429.

23. Boyer MW, Gross TG, Loechelt B, Leemhuis T, Filipovich A, Harris $\mathrm{RE}$. Low risk of graft-versus-host disease with transplantation of CD34 selected peripheral blood progenitor cells from alternative donors for Fanconi anemia. J Pediatr Hematol Oncol 2003; 25: 890895.

24. MacMillan ML, Auerbach AD, Davies SM, Defor TE, Gillio A, Giller $R$, et al. Haematopoietic cell transplantation in patients with Fanconi anaemia using alternate donors: results of a total body irradiation dose escalation trial. $\mathrm{Br} J$ Haematol 2000; 109: 121-129.

25. Enczmann J, Rinker M, Kiesel U, Kuhrober A, Kogler G, Nurnberger $W$, et al. Donor selection process for allogeneic hematopoietic stem cell transplantation at the university hospital of Dusseldorf (19971998). Klin Padiatr 1999; 211: 218-223.

26. Klein T, Yaniv I, Stein J, Narinsky R, Finkelstein Y, Garty BZ. Extended family studies for the identification of allogeneic stem cell transplant donors in Jewish and Arabic patients in Israel. Pediatr Transplant 2005; 9: 52-55.

27. Schipper RF, D'Amaro J, Oudshoorn M. The probability of finding a suitable related donor for bone marrow transplantation in extended families. Blood 1996; 87: 800-804. 